\title{
Varicose Vein Surgery
}

\author{
Michael Ombrellino, M.D., F.A.C.S., ${ }^{1}$ and Lowell S. Kabnick, M.D., F.A.C.S. ${ }^{1}$
}

\begin{abstract}
The treatment of superficial venous disease and chronic venous insufficiency continues to evolve, and the interest in venous disease has matched that in arterial disease in vascular medicine. A better understanding of venous anatomy and pathophysiology and the development of newer, more efficient diagnostic technology have allowed clinicians to utilize minimally invasive techniques in the treatment of varicose veins. These techniques have reduced recurrence and improved overall quality of life (postoperative pain and bruising) following these procedures. This article provides an overview of basic venous surgical anatomy and pathophysiology, along with several older and newer surgical options in the treatment of superficial venous disease. Advantages and disadvantages of each approach are briefly discussed so that the reader may gain better understanding of the options available in the treatment of chronic venous insufficiency.
\end{abstract}

KEYWORDS: Chronic venous insufficiency, varicose veins, neovascularization, endovenous vein obliteration

Objectives: Upon completion of this article, the reader should have a basic understanding of anatomy and pathophysiology as it pertains to several surgical options for superficial venous surgery.

Accreditation: Tufts University School of Medicine (TUSM) is accredited by the Accreditation Council for Continuing Medical Education to provide continuing medical education for physicians.

Credit: TUSM designates this educational activity for a maximum of 1 Category 1 credit toward the AMA Physicians Recognition Award. Each physician should claim only those credits that he/she actually spent in the activity.

The history of venous disease dates back as far as the early beginning of medicine. Early monographs on varicose veins and their surgical treatment were produced as early as $1550 \mathrm{BC} .^{1}$ One of the earliest illustrated descriptions of a varix is found at the base of the Acropolis in Athens and dates to the fourth century BC (Fig. 1). This well-known tablet illustrates a large leg with a tortuous varicosity along its medial aspect. Hippocrates was perhaps the first to recognize the relationship between venous disease and ulceration. ${ }^{2}$ He noted that proper leg elevation and compression allowed faster wound healing and relief of discomfort.

Reports of venous interventions were made as early as 270 BC, in Egypt, with evidence of early varicose vein excision and ligation. ${ }^{3}$ Celsus soon recognized the importance of ligation and division of bleeding varicosities in first century Rome, and Galen, in the second century, devised a method of ligation and vein avulsion using specially designed hooks. ${ }^{4,5}$ These early principles and techniques formed the foundation of what we utilize today in the treatment of superficial venous disease and are the framework of current techniques such as varicose vein stripping and ligation and phlebectomy.

Today, the treatment of venous disease continues to evolve as newer techniques and technology have brought this ancient staple of surgery into the 21st century. With the advent of minimally invasive

Venous Insufficiency; Editors in Chief, Brian Funaki, M.D., Peter R. Mueller, M.D.; Guest Editor, R. Torrance Andrews, M.D. Seminars in Interventional Radiology, volume 22, number 3, 2005. Address for correspondence and reprint requests: Michael Ombrellino, M.D., F.A.C.S., Vein Institute of New Jersey, 95 Madison Avenue, Suite 109, Morristown, NJ 07960. ${ }^{1}$ Vein Institute of New Jersey, Morristown, New Jersey. Copyright (C) 2005 by Thieme Medical Publishers, Inc., 333 Seventh Avenue, New York, NY 10001, USA. Tel: +1(212) 584-4662. 0739-9529,p;2005,22,03, 185,194,ftx,en;sir00311x. 


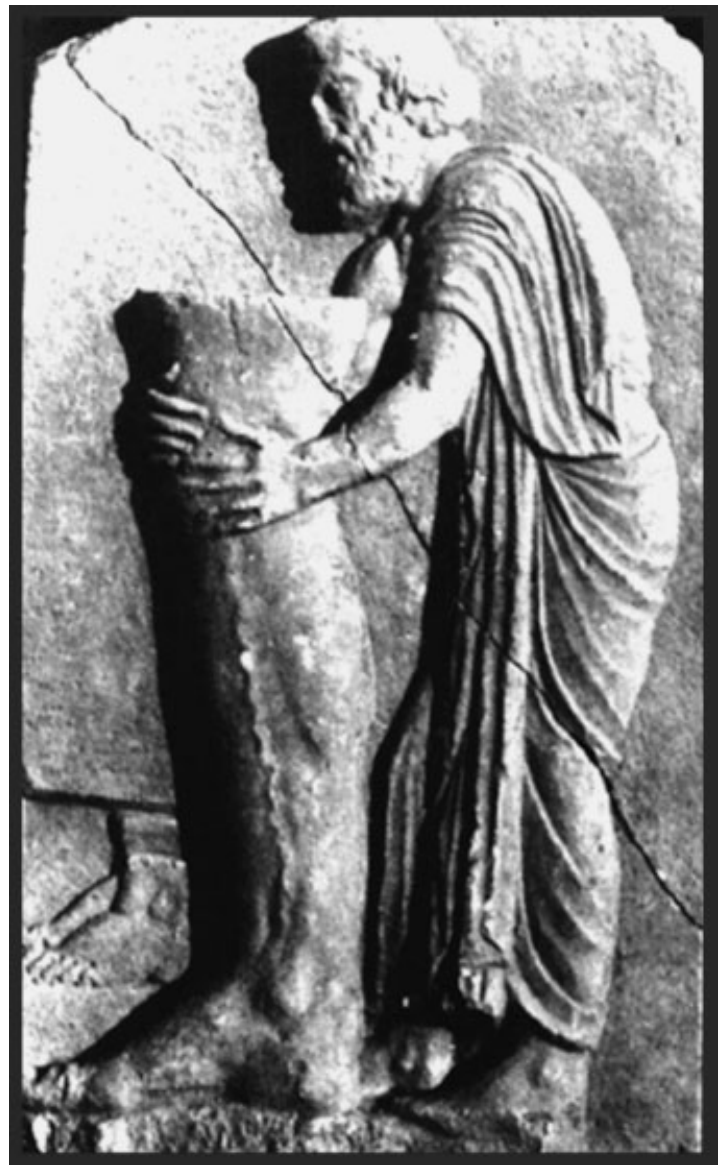

Figure 1 Votive tablet found at the base of the Acropolis in Athens, Greece. It is believed to be the earliest known illustration of a varicose vein.

techniques, improved ultrasound technology, and a multidisciplinary approach, the treatment of superficial venous disease has become a major discipline unto itself. The following paragraphs give an overall view of the indications and several of the most commonly used techniques in the treatment of venous insufficiency, varicose veins, and their sequelae.

\section{EPIDEMIOLOGY AND SYMPTOMOLOGY}

There are several risk factors associated with the development of superficial venous disease and varicosities. A strong family history is perhaps the most common risk factor, ${ }^{6}$ with other factors such as previous history of phlebitis, female gender, pregnancy, and standing for prolonged periods contributing to the development of venous insufficiency and varicosities. ${ }^{7,8}$ Venous insufficiency afflicts both young and old patients with a slight prevalence for the female gender. It is estimated that $\sim 27 \%$ of the adult population in the United States has some form of chronic venous insufficiency, which includes a range of conditions from varicose veins and telangiectasias through chronic skin changes or ulceration, or both. The disease, particularly in the later stages of venous ulceration, impairs overall quality of life and accounts for a significant loss of work time. It has been estimated that in excess of 2 million workdays are lost annually in the United States because of venous ulceration and that $5 \%$ of individuals ultimately lose their jobs because of this disease. ${ }^{9}$ Therefore, it is easy to understand why this disease process has become more prominent in the minds of vascular interventionists and has gained the attention of the American media.

Aside from the late sequel of chronic skin changes (lipodermatosclerosis) and ulceration, the most common signs of chronic venous insufficiency are visible varicosities and telangiectasia. Along with cosmesis, signs and symptoms such as edema, leg fatigue, heaviness, and discomfort are the main reasons for individual patients seeking medical attention. The majority of those treated report significant improvement in their quality of life and an overall improvement in self-esteem.

\section{ANATOMY, PATHOPHYSIOLOGY, AND DIAGNOSIS}

To offer each individual patient an appropriate treatment strategy, it is essential to have a basic understanding of venous anatomy and pathophysiology. A detailed review of venous anatomy and its variations is beyond the scope of this article, and this information can be found in many fine anatomic texts as well as in other articles in this issue.

The axial veins of the lower extremities include the femoral vein (often and incorrectly referred to as the "superficial femoral vein"), the popliteal vein, and the great and short saphenous veins (GSV and SSV). The latter are superficial veins, so designated because they are superficial to the muscular fascia of the leg. The absence of fascial support is often cited as a contributing factor in the valvular incompetence and subsequent reflux in the saphenous system. It is these vessels that are most often addressed in surgery for venous disease.

The GSV originates at the dorsum of the foot, passes anterior to the medial malleolus, and ascends up the medial calf across the posterior-medial aspect of the popliteal space. It then continues up the medial thigh and terminates at the femoral vein at the saphenofemoral junction (SFJ). Clinicians should be aware of basic anatomic variations such as duplicate systems, particularly in the thigh, which, if not identified, can be a source of recurrence and incomplete treatment. Important thigh tributaries of the GSV are the anterior-lateral superficial vein and the posterior-medial superficial vein, which must be preoperatively identified and can be a significant source of reflux and visible varicosities.

The SSV is located posteriorly on the leg; it originates on the lateral aspect of the foot, ascends along the midline of the calf, and terminates into the popliteal vein at variable location in the leg or thigh. The exact 


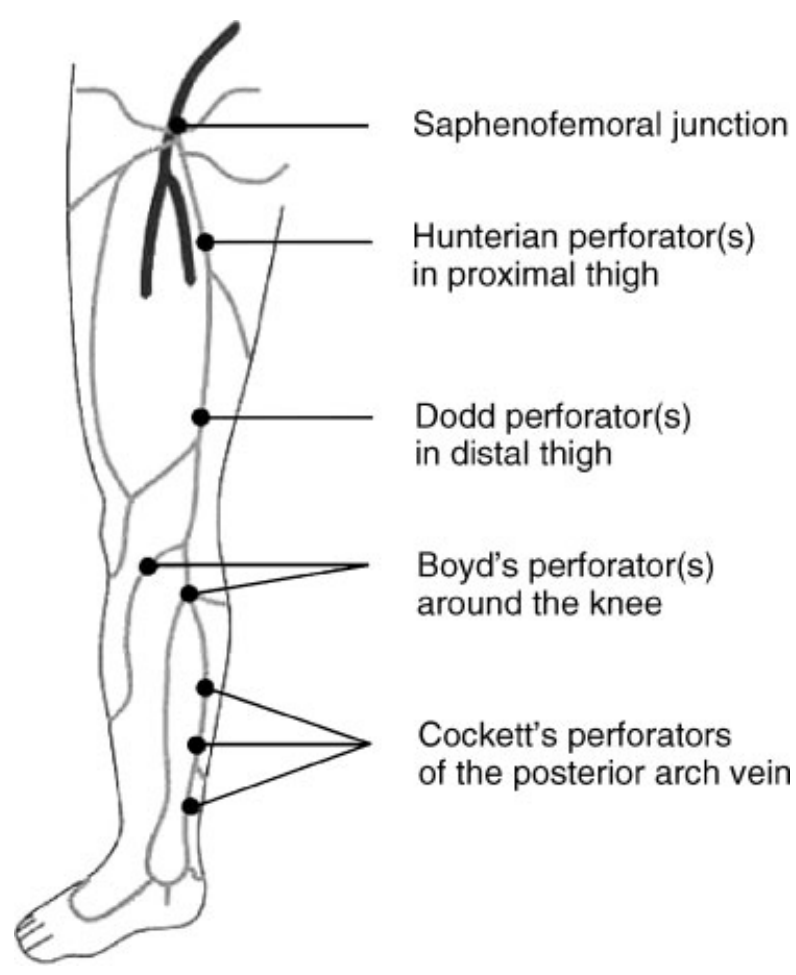

Figure 2 Perforator vein anatomy.

identification of the location of the saphenopopliteal junction (SPJ), as the SSV dips below the fascia, is particularly important when treating reflux originating from this vein. Large, unnamed tributaries originating from this vein can be a source of significant visible varicosities, particularly behind the knee and the calf.

A detailed venous examination must also identify the location and competence of perforating veins (Fig. 2). These are veins that connect the superficial and deep systems either directly, by crossing the fascia, or indirectly, through muscular tributaries. Their presence is particularly important when treating patients with chronic venous ulcers and recurrent varicosities. Although the pattern of perforating veins varies widely among individuals, certain perforating veins are seen commonly enough to be named. These include Cockett's perforators located at the lower leg, Boyd's perforators located at the knee, and Dodd's perforators located along the lower thigh. Burnand et al established the relationship between incompetent communicating perforator veins, varicose veins, and ulcerations. ${ }^{10}$ Reflux in these veins can worsen superficial venous insufficiency and ultimately lead to skin changes and ulcerations. Identification of the perforators and their potential treatment (obliteration) is essential to the overall management of superficial venous disease.

The development of and advances in venous duplex ultrasonography have made this the most essential tool in the diagnosis and treatment of superficial venous disease and valvular reflux. Each practitioner should have a basic understanding of ultrasound imaging as it pertains to venous disease (Fig. 3). It is essential in the identification and diagnosis of anatomic variations and is used to identify the source of venous valvular reflux at its most cephalad point ("point of highest reflux"). Valvular reflux is defined as abnormal-and the valve incompetent-when retrograde flow through the valve lasts longer than 0.5 seconds by duplex criteria. ${ }^{11}$ Reflux should be measured with the patient in an upright position and the limb non-weight bearing. It is imperative that the clinician identify the point of highest reflux to treat the patient properly, that is, the most cephalad point at which valve failure is present. It is also important to identify any large tributary branches of the GSV, SSV, and incompetent perforators to manage the patient adequately.

Much has been written on the topic of recurrence of chronic venous insufficiency and varicose veins following traditional therapeutic options (vein ligation alone or vein ligation and stripping). ${ }^{12}$ Over the past several years, the concept of "neovascularization" has gained increasing popularity and attention. ${ }^{13-16} \mathrm{It}$ is defined as growth and development of new venous
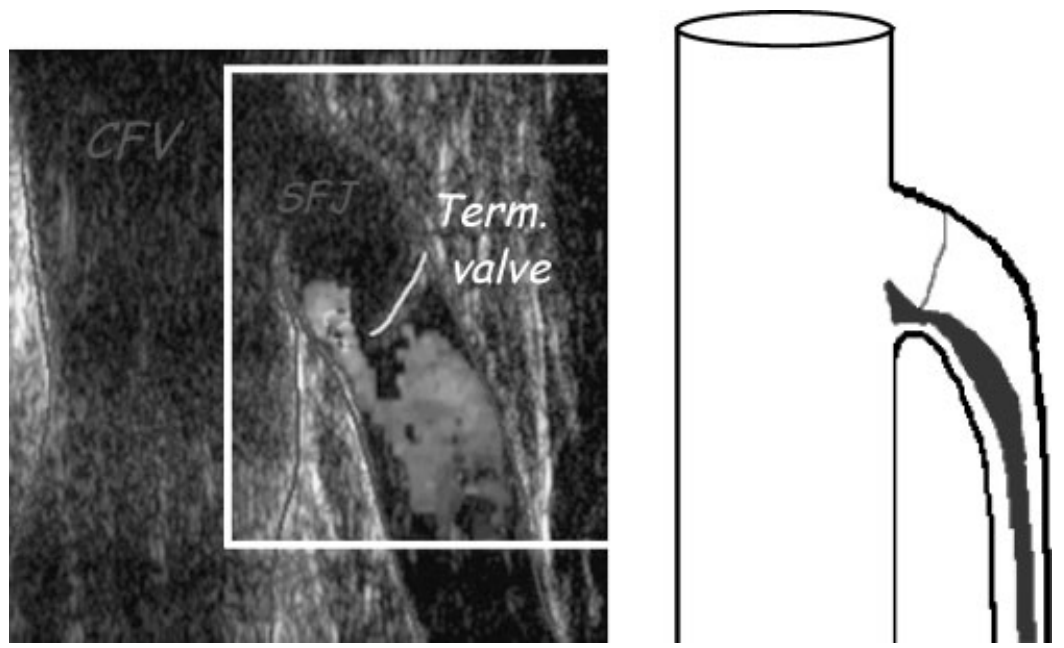

Figure 3 SFJ reflux. Venous duplex ultrasound of the SFJ, demonstrating SFJ reflux and an incompetent terminal valve. 

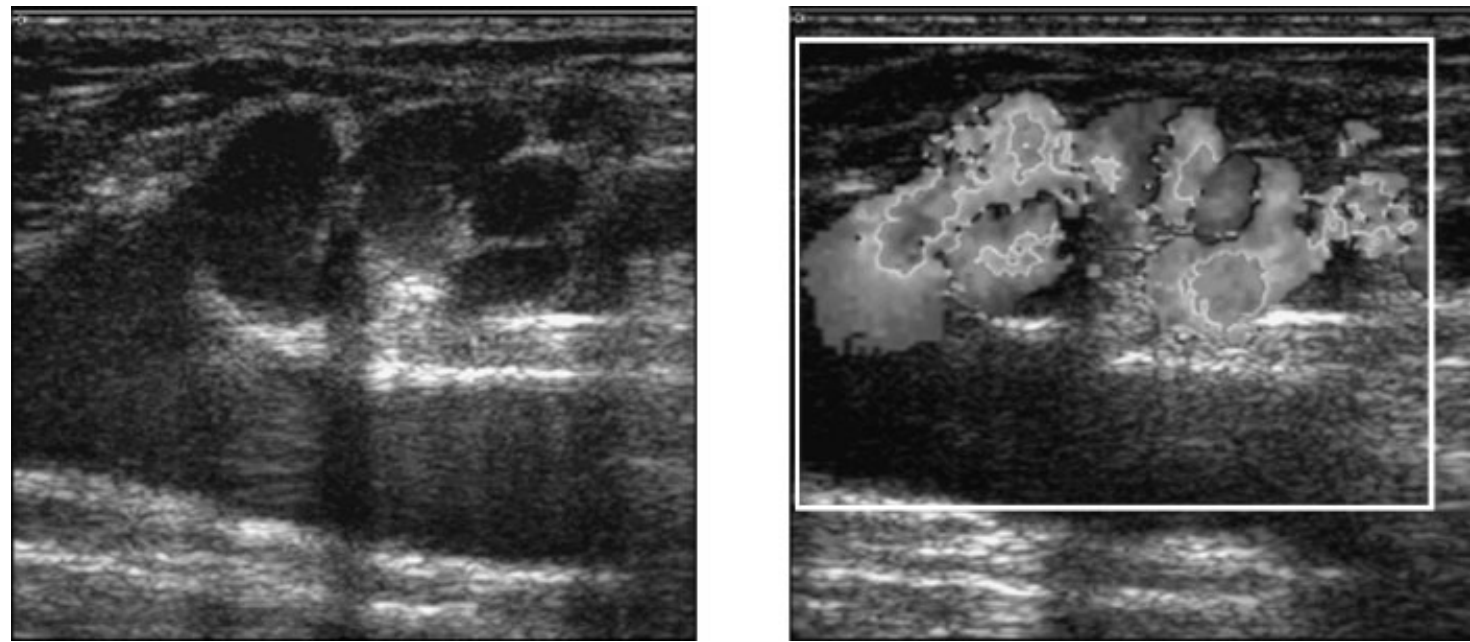

Figure 4 Neovascularization. Venous duplex ultrasound of a symptomatic patient, demonstrating "neovascularization" at the SFJ, 6 years after standard stripping and ligation. (Courtesy of the Vein Institute of New Jersey.)

tributaries (angiogenesis) at the site of previously ligated or stripped varicosities. It is particularly prevalent at the SFJ but may occur at any point of previous vascular surgery (Fig. 4). Its exact prevalence and time of occurrence are difficult to establish because most patients are lost to follow-up. It can manifest itself as long as 10 years after the initial surgery and often leads to recurrence of symptoms and unsightly visible varicosities. It has been postulated that surgical trauma or venous congestion caused by high ligation of venous tributaries of the SFJ may lead to this phenomenon. Although symptom recurrence may, in fact, be a multifactorial event and neovascularization but one of several factors that contribute to the recurrence of venous insufficiency and varicose veins, it stands to reason that avoiding surgical trauma (e.g., groin incisions, skeletonization) improves the likelihood of successful treatment of venous insufficiency. New, minimally invasive techniques such as endovenous obliteration, which avoid a groin incision and closure of SFJ tributaries, seem at this early point in our experience to have a theoretical benefit in this regard. Longitudinal follow-up will be necessary to determine whether this theoretical benefit is truly present.

\section{SURGICAL OPTIONS}

As previously stated, the main indications for seeking treatment of chronic venous insufficiency are symptoms (pain, discomfort, swelling, ulceration, etc.) and cosmesis (visible varicosities) (Table 1). Regardless of the reason, Bergan ${ }^{17}$ suggested that three main objectives be met when planning a surgical treatment strategy for superficial venous disease:

1. Permanent removal of the varicosities along with the source of venous hypertension (highest point of reflux)

2. Obtaining as cosmetic a result as possible
3. Minimizing the number of potential complications

Several procedures available today meet these requirements and are described subsequently.

Despite this general overall approach to venous surgery, the reality is that each procedure must be tailored to meet the anatomic and pathophysiologic needs of the individual patient and should be based upon a detailed venous ultrasound duplex study of the affected limb. The vast majority of varicose vein surgery today is performed on an outpatient or same-day basis, and most patients are able to resume normal activity with relatively little discomfort within a relatively short time period. Many surgical options are available, although some of these have fallen out of favor because of unnecessary potential complications and unacceptably high incidences of recurrence. We describe several of the procedures most commonly performed so that the reader may obtain a basic fundamental knowledge of varicose vein surgery, from both a historical and current prospective.

The surgical options are:

1. Stripping and ligation of the great saphenous vein

2. Varicose vein ligation

3. Phlebectomy

4. Endovenous vein obliteration

5. Perforator vein surgery

Table 1 Treatment of Varicose Veins: Indications for Therapy

\begin{tabular}{ll}
\hline Cosmesis & Bleeding \\
Leg pain & Lipodermatosclerosis \\
Leg heaviness & Venous ulceration \\
Leg fatigue & Dermal hyperpigmentation \\
\hline
\end{tabular}




\section{Stripping and Ligation of the Great Saphenous Vein}

Until recently, ankle-to-groin stripping of the GSV with ligation of branch tributaries was considered the "gold standard" in varicose vein surgery. However, with a better understanding of both anatomic relationships and associated venous physiology, this excessively radical procedure has, for the most part, been abandoned. It is now recognized that stripping of the GSV to the level of the knee is sufficient to obtain optimal results and avoids the troublesome complication of saphenous nerve injury associated with stripping in the calf. ${ }^{18}$ The practice of ligating and disconnecting each saphenous vein tributary in the groin (a process called skeletonization) is now believed to contribute to venous congestion and angiogenesis, resulting in SFJ neovascularization and recurrence, and has also fallen out of favor.

The most popular current technique for stripping (more appropriately called saphenectomy) utilizes a disposable, flexible, plastic internal stripper (i.e., Codman stripper) (Fig. 5). A small transverse incision is made along the skin creases at the groin. The GSV is isolated at the SFJ and ligated at the junction. A venotomy is then made and the stripper is passed into the GSV at the groin and threaded caudally through the incompetent vein caudal to the level of the knee, where it is brought out through a small skin incision and externalized. After the vein has been transected at the SFJ and at the knee, an acorn-shaped stripping head is attached to the stripper and the entire device drawn caudally. The stripping head prevents the stripper from passing through the end of the vein, which is instead inverted and pulled through the thigh, tearing away from its branches as it proceeds. Both incisions are then closed and a compression dressing applied. This procedure is usually done under general anesthesia, although advances in tumescent anesthesia have allowed some clinicians to avoid the use of general anesthesia. $^{19}$

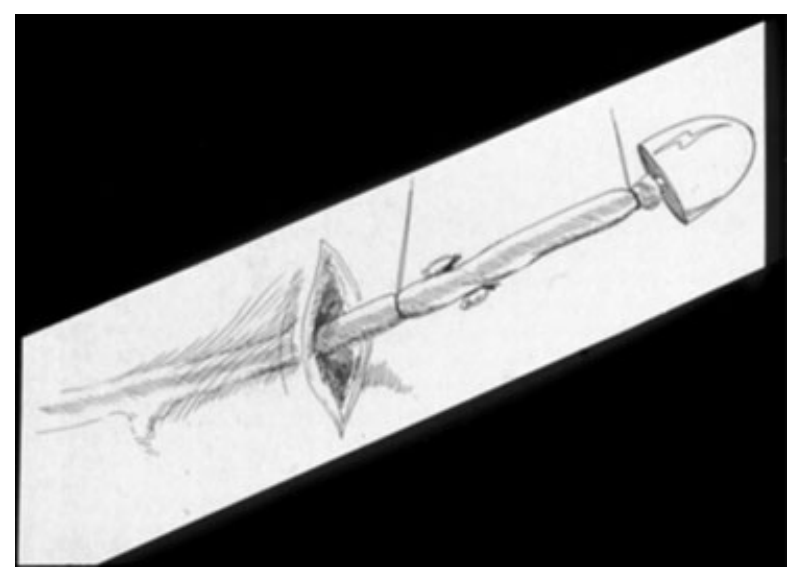

Figure 5 Codman Stripper. After exposing the GSV, a flexible plastic stripper is inserted within the vein and subsequently pulls out through a separate incision.
Proponents of this procedure point to the fact that complete saphenectomy eliminates any possibility of persistent axial reflux through the segment of the vein removed. Winterborn et al demonstrated a $60 \%$ reduced risk of reoperation at 11 years after ligation and stripping. ${ }^{16}$ Opponents of this procedure cite the increased postoperative pain, bruising, and longer overall recovery time resulting in decreased overall quality of life. Neovascularization, particularly at the groin, has also been associated with traditional stripping and may increase the possibility of recurrences. ${ }^{13}$ Despite this potential for eventual occurrence, stripping and ligation remain a viable option in the treatment of saphenous vein disease.

\section{Varicose Vein Ligation Only}

Popularized in the 1970s and 1980s, the concept of ligating visible varicosities utilizing multiple incisions has fallen out of favor as both ineffective and cosmetically unacceptable. Multiple small incisions are made along the course of visible varicosities and the veins are segmentally ligated and cut. With the current understanding that venous insufficiency must be addressed at its point of highest reflux, it is easy to understand why simple ligation of visible varicosities is bound to fail. Only after correcting the origin of highest reflux in the axial vein can secondary visible varicosities be ligated and removed. Failure to do so results in predictably early local recurrences at the sites adjacent to the previous incisions. ${ }^{15}$ Sarin et al have shown a recurrence rate as high as $45 \%$ after ligation alone as early as 3 months after treatment. $^{20}$ Dwerryhouse et al reported a recurrence rate of $71 \%$ after high ligation alone. ${ }^{14}$ The majority of the clinicians who perform venous surgery have therefore mostly abandoned varicose vein ligation as an isolated procedure.

Ligation of the SSV at the SPJ through a small skin incision is still performed in the presence of varicosities originating from the SSV. Preoperative venous ultrasound mapping of the junction is imperative to perform this procedure properly and safely. Intraoperative identification of the sural nerve, which lies in close proximity to the vein at the junction, is important in avoiding injury to this nerve. Ligation of the vein at the junction addresses the underlying problem of reflux at the most cephalad point (SPJ). This procedure is usually combined with an extensive phlebectomy to eliminate visible varicosities.

\section{Phlebectomy}

First described by Cornelius Celsus in the first century $\mathrm{AD}$ and perfected in modern Europe, phlebectomy is today an essential part of the armamentarium of venous surgery. ${ }^{21}$ Phlebectomy of varicose veins may 
be performed alone or in combination with other vein procedures. Referred to in the past as "stab avulsion phlebectomy" and today as "microphlebectomy," this ancient procedure has undergone significant refinements and a newfound renaissance over the last several years. The procedure involves utilizing a small blade (11 scalpel, 18-gauge needle, or small ophthalmologic blade) to make a cutaneous microincision. Incisions should generally be vertically oriented along the thigh and lower leg and should follow the dermal lines at the knees and ankles to obtain optimal cosmetic results. Phlebectomy hooks (Muller, Oesch, Varady, etc.) are used to pick up the vein and bring it through the incision, where it is grasped, transected, and avulsed (Fig. 6). The use of the small blades allows cosmetically acceptable results and allows resumption of normal activity within a relatively short period of time. Microphlebectomy is appropriate after other venous procedures (stripping and ligation or endovenous obliteration) when established visible varicose saphenous tributaries or clusters are associated with incompetent perforating veins. ${ }^{22}$

Recently, a technique known as power phlebectomy (TriVex ${ }^{\mathrm{TM}}$ ) has gained favor with some clinicians. It involves the use of a transilluminated power phlebectomy device that macerates and extracts visualized veins through a small incision site. The technique is particularly effective when dealing with a large area of vein clusters and significantly reduces operative time. Advances in tumescent anesthesia have reduced the postoperative pain associated with this procedure, although bruising still remains an issue.

The results of phlebectomy are excellent when the procedure is performed for the appropriate indications and when, as stated before, more cephalad reflux is also addressed. Performing this procedure without addressing an underlying reflux problem may offer satisfactory short-term results but ultimately leads to early recurrence and an unhappy patient. Phlebectomy procedures can be performed in an ambulatory setting, utilizing local anesthetic, tumescent anesthesia, and/or light sedation. They can be done at the same setting as other vein procedures, be performed as the second part of a staged procedure, or be done as a stand-alone procedure for recurrent veins. As with any form of treatment for varicose veins, new varicosities may develop over time and patients should be informed of this potential.

\section{Endovenous Vein Obliteration}

In the age of minimally invasive surgery, endovenous vein obliteration is quickly gaining popularity among clinicians and patients alike. It offers a highly effective and cost-effective alternative to traditional stripping and ligation, utilizing one of two distinct forms of thermal energy - radiofrequency $(\mathrm{RF})$ and laser — to destroy the vein in situ endovascularly and eliminate the highest point of reflux. Most frequently, it is utilized to treat axial vein reflux (GSV and SSV), but many clinicians have expanded its use to large venous tributaries. Both modalities rely heavily on the use of tumescent anesthesia to insinuate fluid between the skin and the vein under treatment, thereby reducing the potential for thermal damage to the skin. The use of intraoperative ultrasound is essential in completing the procedure.

$\mathrm{RF}$ endovenous vein obliteration involves the use of a $460-\mathrm{kHz}, 25-\mathrm{W}$ generator with a specially devised disposable electrode catheter to deliver bipolar RF energy to the vein. This accomplishes controlled heating $\left(85^{\circ} \mathrm{C}\right)$ of the vessel wall, causing collagen destruction and contraction, which results in obliteration of the vein. By limiting the temperature of the electrodes in contact with the vessel wall to less than $100^{\circ} \mathrm{C}$,

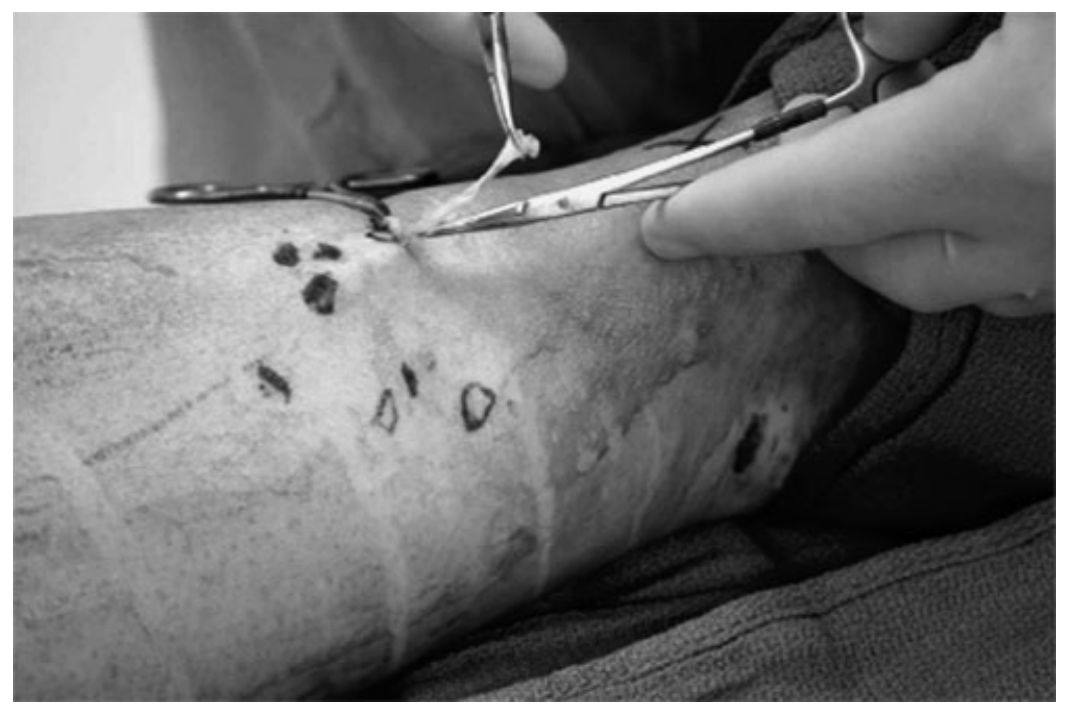

Figure 6 Microphlebectomy. Visible varicosities are preoperatively marked. A small blade is used to make a micro-incision and hooks and clamps are used to avulse the vein through the incisions. (Courtesy of the Vein Institute of New Jersey.) 
boiling, vaporization, and carbonization of the tissue are avoided. One such device on the market is the Closure ${ }^{\circledR}$ catheter system (VNUS Medical Technologies, Sunnyvale, CA). It is approved by the Food and Drug Administration (FDA) and widely available in the United States for the treatment of GSV reflux. It is available in two sizes -5 and $8 \mathrm{Fr}-$ to reflect the diameter of the vein being treated (Fig. 7). Early results have been promising, and both patients' satisfaction and quality of life are improved after this procedure even at 2 years. ${ }^{23}$ Opponents of RF cite a higher learning curve and the higher cost of this procedure when compared with standard stripping and ligation and with vein obliteration using laser energy.

Endovenous laser vein obliteration utilizes one of four different wavelengths (810, 940, 980, and recently $1320 \mathrm{~nm}$ ) to destroy the lining of the vein wall endothermally and thereby cause vein wall contraction and obliteration. Wavelength does not seem to influence the efficacy of treatment, although anecdotal evidence suggests that higher wavelengths may reduce postprocedural bruising and pain. There are several manufacturers in the United States who distribute these devices, which are FDA approved for endovenous ablation of axial varicose veins. Lower overall cost and significantly faster procedure times (versus RF ablation) make this modality particularly appealing to some clinicians.

Procedural techniques are similar with both modes of endovenous vein obliteration. Patients are

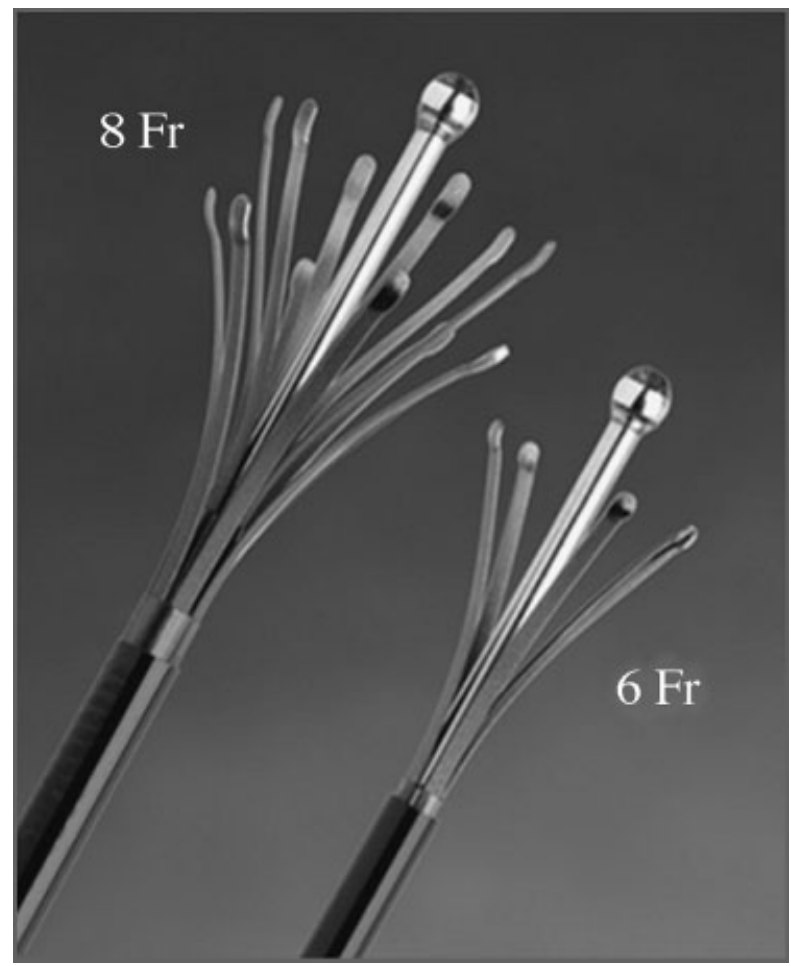

Figure 7 RF electrode. Electrodes are available in two sizes depending on the size of the vein to be treated. (Courtesy of VNUS Medical Technologies, Sunnyvale, CA.) preoperatively vein mapped using duplex ultrasound. Local anesthetic is used at the puncture site, which is often at or just below the knee. Utilizing duplex guidance, an entry needle is inserted into the saphenous vein (Fig. 8). Once entry is established with confirmation of venous return from the needle, a wire is advanced under ultrasound guidance into the vein. A vascular sheath is then inserted over the wire and positioned $\sim 2 \mathrm{~cm}$ caudal to the SFJ. A catheter electrode (RF) or laser fiber is placed through the sheath to a point just caudal to the SFJ (Fig. 9). Tumescent local anesthesia (0.15-0.25\% diluted and buffered lidocaine) is then infiltrated around the entire length of the vein to be treated. A pullback technique is then used to ablate the vein endothermally. Typical pullback rates are $2-3 \mathrm{~cm} / \mathrm{min}$ with $\mathrm{RF}$ and 10 $12 \mathrm{~cm} / \mathrm{min}$ with laser. At the termination of the procedure, an intraoperative ultrasound examination is done at the SFJ to rule out a deep venous thrombosis (DVT) and a compressive dressing (class 2) is applied. The patients are sent home following a brief recovery period.

Patients are encouraged to resume normal daily activity as soon as possible and are seen in follow-up 2472 hours postoperatively. A venous duplex examination is performed at the postoperative visits to confirm vein obliteration and rule out DVT. At our institution, follow-up venous duplex studies are done at 4 months and 1,2 , and 3 years. It has been our experience that if the vein remains closed at 4 months, the GSV will remain closed, fibrosed, and unrecognizable from the surrounding tissue at 1 year.
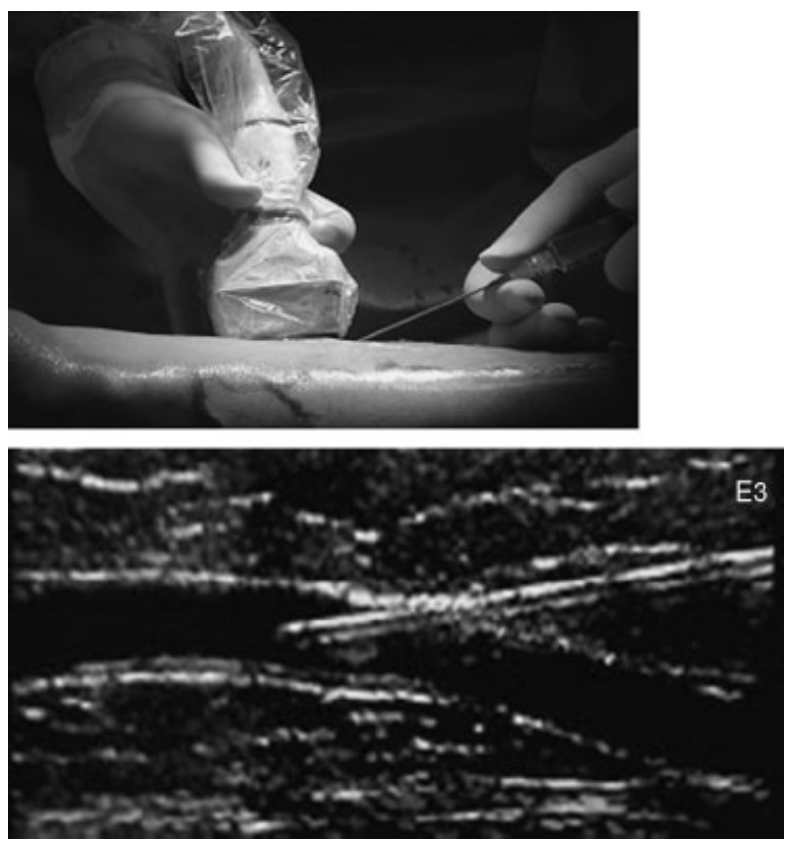

Figure 8 Ultrasound guided entry into the GSV. An entry needle is placed into the GSV at the knee, under ultrasound guidance, and entry is confirmed by aspirating blood into the syringe. (Courtesy of the Vein Institute of New Jersey.) 


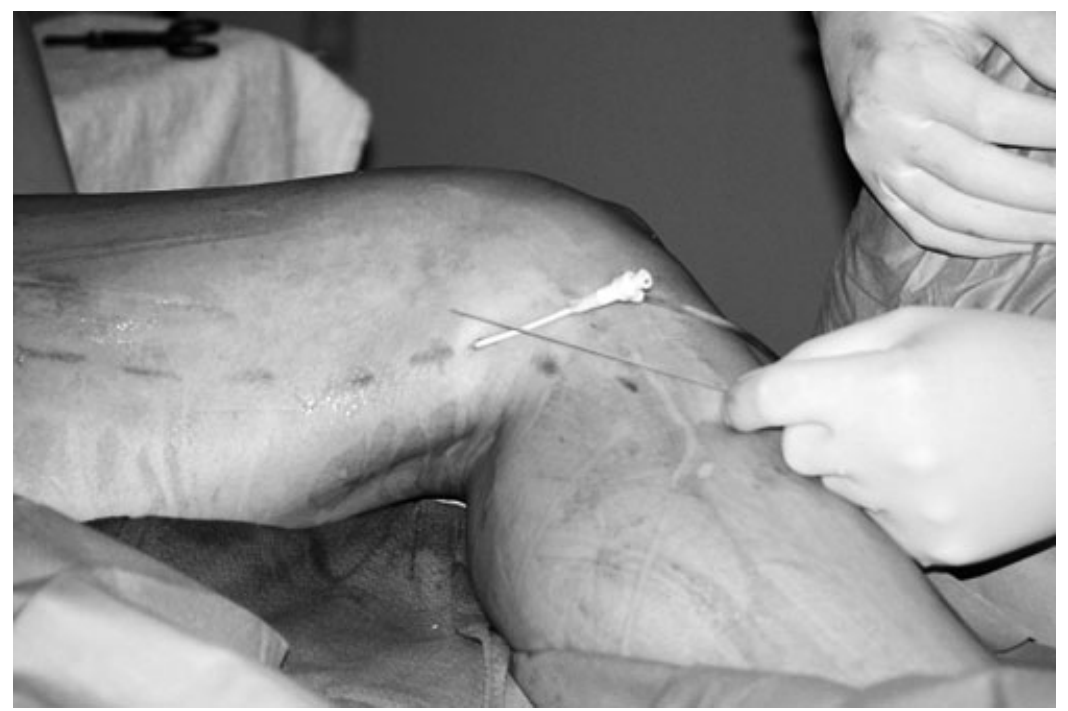

Figure 9 Endovenous sheath and laser fiber. Once entry into the vein is established, a 6 French sheath is inserted into the vein over a wire and the laser fiber placed into the sheath. Both sheath and fiber should be at least $2 \mathrm{~cm}$ proximal to the SFJ. (Courtesy of the Vein Institute of New Jersey.)

Although endovenous obliteration is a relatively safe procedure, the performing clinician must be aware of several unwanted postprocedure complications and results. There have been reports of DVT following this procedure. ${ }^{24,25}$ This can easily be avoided by remaining at least $2 \mathrm{~cm}$ from the SFJ utilizing ultrasound confirmation. In the event that a DVT does occur, it should be treated accordingly with anticoagulation therapy and close clinical follow-up for progression or regression with venous duplex ultrasound. Paresthesia and thermal skin injury have been reported in several patients undergoing endovenous obliteration. These can easily be avoided with utilization of an adequate amount of tumescent anesthesia to insulate the surrounding tissue from the vein being treated. It is recommended that a cuff of at least $1 \mathrm{~cm}$ of tumescent anesthesia around the vein be infiltrated to avoid these complications. If paresthesia does occur, it is typically self-limiting and usually resolves in several months. Bruising and postoperative pain, although significantly less than those found with standard stripping and ligation, can occur but are usually well tolerated and should resolve completely in several days. As stated earlier, it seems that higher wavelengths can reduce these symptoms. Persistence of pain and swelling warrants further investigation such as duplex ultrasound to exclude the possibility of DVT. Finally, failure of the vein to close is sometimes encountered and is believed to occur when the amount of tumescent anesthesia used is less than that necessary to compress large-diameter veins against the energy source at initial treatment. These veins can usually be retreated following a short recovery period. Despite these infrequent complications and events, endovenous vein obliteration is quickly becoming the treatment of choice among clinicians and patients as a minimally invasive alternative to varicose stripping and ligation.

Results with endovenous obliteration have thus far been good (Fig. 10). In a multicenter study involving 286 patients and 319 limbs treated utilizing RF, 83.6\% and $85.2 \%$ of the limbs treated remained closed at 12 and 24 months, respectively. The failure rate (recanalization) was $10.8 \%$ at 12 months and $11.3 \%$ at 24 months. There was a significant reduction in visible varicosities and patients' satisfaction was achieved in $92 \%$ at 1 year and $94.5 \%$ at 2 years. $^{26}$ The results utilizing laser vein obliteration, although not as extensively published, appear to be just as good. ${ }^{27}$ Certain modifications of the procedure have significantly reduced the failure rates since the early trails. ${ }^{23}$

\section{Perforator Vein Surgery}

Incompetent perforating veins are often seen in patients with chronic venous ulcerations, and understanding of their treatment is important to any clinician treating these patients. ${ }^{28}$ Compression stockings (class 2 and 3) and proper wound care are often sufficient to heal these troublesome wounds. ${ }^{29,30}$ In the event that these ulcers do not heal or are frequently recurrent, primary perforator insufficiency or post-thrombotic syndrome can often be established as a contributing factor. In such situations, several surgical options are available. Liton's original operative technique, described in the early 1950s, involved surgical ligation of subfascial perforators through three long skin incisions along the medial, anterolateral, and posterolateral calf. This radical procedure has since been mostly abandoned because of its high rate of wound complications. Subsequent 

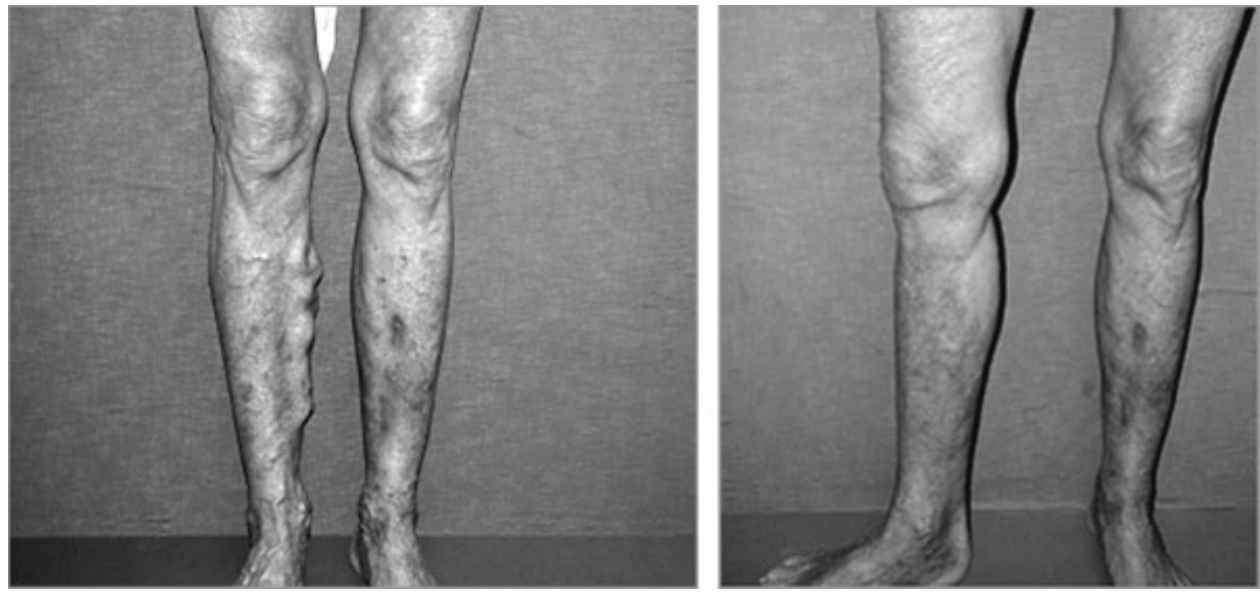

Figure 10 Results of endovenous laser. Preoperative picture of extensive varicosities and improved cosmetic results 4 months after endovenous laser obliteration. (Courtesy of the Vein Institute of New Jersey.)

authors, including Cockett and DePalma, modified the procedure utilizing smaller, more strategically placed incisions, combined with excision of axial superficial vein and placement of skin grafts. Despite these modifications, wound complications were still prevalent and better alternatives were sought.

In the hope of eliminating wound complications, Edwards devised a device called a phlebotome, which is introduced through a small medial skin incision just below the knee and advanced subfascially toward the medial malleolus, disrupting the perforators blindly as it is advanced. The advantage of the technique is that the incision is remote from the skin being compromised by venous disease, which reduces local complications. Its disadvantage is the blind nature of branch disruption. With these issues in mind, subfascial endoscopic perforator surgery (SEPS) was born. During SEPS, instruments are introduced into the subfascial space through two or fewer small, remote incisions and each perforator is identified, clipped, and divided with endoscopic visualization. Because this procedure is often combined with concomitant saphenous vein ablation, the isolated value of SEPS is difficult to assess.

Early results of perforator vein surgery were promising, but recurrence of venous ulcers years after the procedure has lately become an issue. This has been particularly true with ulcer patients who presented with post-thrombotic syndrome as their underlying factor. ${ }^{31-34}$ Preliminary results with the use of sclerotherapy under ultrasound guidance are promising and this may one day supplant SEPS as the procedure of choice for local control of perforator incompetence.

\section{SUMMARY}

With new insights into the anatomy, pathophysiology, and progression of venous disease and with recent technical advances, the treatment of superficial venous disease has gained its rightful place in the realm of vascular medicine. The concept of initially treating the highest point of reflux is now widely accepted among clinicians who perform venous procedures and has drastically improved overall long-term results. With the use of emerging technologies and an ever-present consumer demand, this once overlooked discipline of vascular medicine has come to the forefront as a specialty. The use of minimally invasive techniques such as endovenous vein obliteration has opened this field to multiple specialties and has significantly increased the quality of life of the patients undergoing these procedures. The next challenges for clinicians who treat venous disease are those of limiting recurrence and-the last frontier of venous disease - the effective correction of deep venous insufficiency. As newer technology and a better understanding of our current failures progress, we may ultimately resolve these pending issues.

\section{REFERENCES}

1. Majno G. The Healing Hand. Cambridge, MA: Harvard University Press; 1975

2. Hippocrates. The Genuine Works of Hippocrates. Adams EF, trans. Vol 2. New York: Wm Wood \& Co; 1886

3. Anning ST. Historical aspects. In: Dodd H, Cockett FB, eds. The Pathophysiology and Surgery of Veins of the Lower Limb. 2nd ed. Edinburgh: Livingstone; 1976:3-17

4. Celsus AC. Of Medicine in Eight Books. Grieve J, trans. London: Wilson \& Durham; 1756

5. Walsh J. Galen's writing and influences inspiring them. Ann Med Hist 1934;I:14

6. Jamieson WG. State of the art of venous investigation and treatment. Can J Surg 1993;36:119-128

7. Brand FN, Dannenberg AL, Abbott RD, Kannel WB. The epidemiology of varicose veins: the Framingham study. Am J Prev Med 1988;4:96-101

8. Scott TE, LaMorte WW, Gorin DR, Menzoian JO. Risk factors for chronic venous insufficiency: a dual case-control study. J Vasc Surg 1995;22:622-628 
9. Browse NL, Burnand KG, Thomas ML. Diseases of the Veins: Pathology, Diagnosis and Treatment. London: Hodder and Stoughton; 1988

10. Burnand KG, O’Donnell TF, Thomas ML, Browse NL. The relative importance of incompetent communicating veins in the production of varicose veins and venous ulcers. Surgery 1977;82:9-14

11. Vasdekis SN, Clarke GH, Nicolaides AN. Quantification of venous reflux by means of duplex scanning. J Vasc Surg 1989; 10:670-677

12. Royle JP. Recurrent varicose veins. World J Surg 1986;10: 944-953

13. Jones L, Braithwaite BD, Selwyn D, Cooke S, Earnshaw JJ. Neovascularization is the principal cause of varicose vein recurrence: results of a randomized trial of stripping the long saphenous vein. Eur J Vasc Endovasc Surg 1996;12:442-445

14. Dwerryhouse S, Davies B, Harradine K, Earnshaw JJ. Stripping the long saphenous vein reduces the rate of reoperation for recurrent varicose veins: five-year results of a randomized trail. J Vasc Surg 1999;29:589-592

15. Glass GM. Neovascularization in recurrence of varices of the great saphenous vein in the groin: phlebography. Angiology 1988;39:577-582

16. Winterborn RJ, Foy C, Earnshaw JJ. Causes of varicose vein recurrence: late results of a randomized controlled trail of stripping the long saphenous vein. J Vasc Surg 2004;40:634639

17. Bergan JJ. Varicose veins: treatment by surgery and sclerotherapy. In: Rutherford RB, ed. Vascular Surgery. 5th ed. Philadelphia: WB Saunders; 2000:2011-2012

18. Bergan JJ. Surgery of the Veins of the Lower Extremity. Philadelphia: WB Saunders; 1985

19. Proebstle TM, Paepcke U, Weisel G, Gass S, Weber L. High ligation and stripping of the long saphenous vein using tumescent technique for local anesthesia. Dermatol Surg 1998;24:149-153

20. Sarin S, Scurr JH, Coleridge Smith PD. Assessment of stripping of the long saphenous vein in the treatment of primary varicose veins. Br J Surg 1992;79:889-893

21. Olivencia JA. Ambulatory phlebectomy turned 2400 years old. Dermatol Surg 2004;30:704-708
22. Kabnick LS. Should we consider a paradigm shift for the treatment of GSV and branch varicosities? 2003 UIP World Congress Phlebology Chapter Meeting, San Diego

23. Manfrini S, Gasbarro V, Gudmundur D, et al. Endovenous management of saphenous vein reflux. J Vasc Surg 2000;32: 330-342

24. Merchant RF, DePalma RG, Kabnick LS. Endovascular obliteration of saphenous reflux: a multicenter study. J Vasc Surg 2002;35:1190-1196

25. Min RJ, Khilnani NM. Endovenous laser treatment of saphenous vein reflux. Tech Vasc Interv Radiol 2003;6:125131

26. Komenaka IK, Nguyen ET. Is there an increased risk for DVT with the VNUS closure procedure? J Vasc Surg 2002; 36:1311

27. Hingorani AP, Ascher E, Markevich N, et al. Deep venous thrombosis after radiofrequency ablation of the greater saphenous vein: a word of caution. J Vasc Surg 2004;40: 500-504

28. Delis KT. Perforator vein incompetence in chronic venous disease: a multivariate regression analysis model. J Vasc Surg 2004;40:626-633

29. Negus D. Prevention and treatment of venous ulcerations. Ann R Coll Surg Engl 1985;67:144-148

30. Mayberry JC, Moneta GL, Taylor LM, Porter JM. Fifteenyear results of ambulatory compression therapy for chronic venous ulcers. Surgery 1991;109:575-581

31. Burnand K, Thomas ML, O’Donnell T, Browse NL. Relation between post-phlebitic changes in the deep veins and the results of surgical treatment of venous ulcers. Lancet 1976;1: 936-938

32. Johnson WC, O'Hara ET, Corey C, Widrich WC, Nabseth DC. Venous stasis ulceration: effectiveness of subfascial ligation. Arch Surg 1985;120:797-800

33. Robison JG, Elliot BM, Kaplan AJ. Limitations of subfascial ligation for refractory chronic venous stasis ulceration. Ann Vasc Surg 1992;6:9-14

34. Gloviczki P, Cambria RA, Rhee RY, Canton LG, McKusick MA. Surgical technique and preliminary results of endoscopic subfascial division of perforating veins. J Vasc Surg 1996;23: $517-523$ 